\title{
Treatment with Oral Appliances in Obstructive Sleep Apnea
}

\author{
Palak Srivastava $^{1, *}$, Laishram Memory devi ${ }^{1}$, Vatsal Chauhan ${ }^{2}$ \\ ${ }^{1}$ Dental Practitioner \\ ${ }^{2}$ Department of Conservative Dentistry and Endodontics Peoples college of dental sciences and research centre
} *Corresponding author: Palak Srivastava, Dental Practitioner

Received date: 26 November, 2021 | Accepted date: 08 December, 2021 |

Published date: 10 December, 2021

Citation: Srivastava P, Devi LM, Chauhan V. (2021) Treatment with Oral Appliances in Obstructive Sleep Apnea. J Dent Oral Epidemiol 1(2). doi https://doi.org/10.54289/JDOE2100108

Copyright: () 2021 Srivastava P, et al. This is an open-access article distributed under the terms of the Creative Commons Attribution License, which permits unrestricted use, distribution, and reproduction in any medium, provided the original author and source are credited.

\begin{abstract}
Obstructive sleep apnea (OSA) is a disorder caused by a number of factors like an obstruction of the upper airway during sleep because of insufficient motor tone of the tongue and/or airway dilator muscles or inadequate growth of the maxillary jaw bone etc. Oral appliances (OAs) are commonly used as a non-invasive treatment for obstructive sleep apnea syndrome. The primary oral appliance (OA) used in obstructive sleep apnea (OSA) treatment is the mandibular advancement device (MAD). Tongue-retaining devices or tongue-stabilizing devices (TSDs) are a second type of OA, which displace the tongue anteriorly and may be customized or come in different stock sizes. This review article aims to examine the best in class on this particular subject of treatment of OSA with oral appliances, explaining acceptability of an appliance in patients on the basis of its construction and results, while providing enough cognizance regarding the diagnosis, management and causes of discontinuation.

Abbreviations: OSA: Obstructive sleep apnea, OA: oral appliance, MAD: mandibular advancement device, TSD: tonguestabilizing devices, PSG: polysomnography, OCST: out-of-center sleep testing, HSAT: Home sleep apnea testing, EEG: electroencephalography, AASM: Academy of Sleep Medicine, CBCT: Cone beam computed tomography.
\end{abstract}

\section{Introduction:}

What is obstructive sleep apnea? Obstructive sleep apnea (OSA) is a disorder during which an individual frequently stops breathing during his or her sleep. It results from an obstruction of the upper airway during sleep that happens due to inadequate motor tone of the tongue and/or airway dilator muscles [1]. In addition to accelerated mortality, different negative health effects related to untreated OSA consist of cardiovascular disease, cerebrovascular events, diabetes, and cognitive impairment. Additionally, OSA is negatively related to quality of lifestyles and definitely related to motor automobile accident injuries [2]. The field of orthodontia plays an important role in the diagnosis and treatment of sleep-disordered breathing syndromes. Oral appliances have been used to treat mild-to moderate obstructive sleep apnea [3].

\section{Diagnosis}

In the joint assessment and treatment of patients with OSA using oral appliances, dentists were involved. Dentists play a key role in determining the suitability of OAT in patients with OSA, selecting the right OAT device, modifying the OAT device, and testing the patient for adverse effects.

Primarily OSA is considered as a medical condition because 
of the several co-morbidities related with it such as heart failure and asthma, and other sleep disorders such as insomnia, parasomnias and periodic limb. It is important that dentists work collaboratively with the referring sleep medicine physician to perform these tasks. If this happens, patients will have the best chance for their OSA to be handled efficiently movements [4].

\section{IN ADULTS}

Diagnostic confirmation of OSA is performed by asleep medicine specialist with the use of the gold standard of an incenter overnight sleep study (polysomnography [PSG]) or out-of-center sleep testing (OCST) for appropriately selected patients. Home sleep apnea testing (HSAT) is a type of OCST. Attended PSG includes a minimum of 7 channels of recording, including electroencephalography (EEG), electrocardiography, and monitoring of sleep, airflow through the nose and mouth, pulse oximetry, respiratory effort, and leg movement. HSAT includes 4 - 7 channels. It is important to notice that HSAT typically does not include EEG monitoring of sleep.

OSA are often diagnosed by either of two sets of criteria. The first set of diagnostic criteria for OSA includes the presence of at least 1 of the following:

(1) The patient has sleepiness, non-restorative sleep, fatigue, or insomnia symptoms

(2) The patient wakes with breath holding, gasping, or choking

(3) A bed partner or other observer reports habitual snoring, breathing interruptions, or both during the patient's sleep, and (4) The patient has been diagnosed with hypertension, a mood disorder, cognitive dysfunction, coronary artery disease, stroke, congestive heart failure, atrial fibrillation, or type2 diabetes mellitus.

In the second criteria, OSA are often diagnosed if PSG or OCST shows 15 or more predominantly obstructive events (obstructive or mixed apneas, hypopneas, or RERAs per hour of sleep during a PSG or per hour of monitoring on OCST) [5].

Academy of Sleep Medicine (AASM) classifies OSA severity in according to AHI as mild (5 - 15 events per hour), moderate (> 15 - 30events per hour), or severe (> 30 events per hour) [6].
The AHI is a coarse and inaccurate parameter and any measure of OSA severity should capture the pathophysiologic diversity of the disease phase [7]. Since only the rate of events is recorded, AHI integrates intensity of the individual events only to the degree that event severity correlates with frequency. What is required today is not to throw out the AHI entirely but, rather, to demote it from the level of "goldstandard" as a severity metric and use it rationally [8].

Orthodontic radiographs: Cone beam computed tomography (CBCT) have been demonstrated to be helpful in morphometric investigation of the hard and delicate tissues for diagnosis in routine orthodontic treatment, however they have certain constraints in regards to the determination of OSA. There is as of now no insignificant cross-sectional region or volume of the airway that has been approved as a threshold limit at which an individual is at higher danger of having OSA. Along these lines, orthodontic records might be taken by the orthodontist, however at present no radiographic strategies have been accounted for to have sufficiently high affectability or particularity to fill in as a danger evaluation instrument for OSA [5].

\section{IN CHILDREN}

Analysis of OSA in youngsters is affirmed exclusively by the highest quality level PSG. Along with standard recording channels, all paediatric PSG is presently led with $\mathrm{CO} 2$ observing. Estimation with either end-flowing $\mathrm{CO} 2$ (the partial pressure of $\mathrm{CO} 2$ present toward the finish of exhalation) or transcutaneous $\mathrm{CO} 2$ check is satisfactory.

As per the International Classification of Sleep Disorders, OSA can be analyzed by both of 2 arrangements of demonstrative measures. The original set of standards for OSA incorporates the presence of in any event 1 of the accompanying: (1) snoring, (2) labored, paradoxic, or obstructed breathing during the child's sleep, or (3) sleepiness, hyperactivity, behavioural problems, or learning problems; and polysomnography shows one or more obstructive apneas, mixed apneas, or hypopneas per hour of sleep.

On the other hand, OSA can be analyzed if the PSG shows an event of obstructive hypoventilation, which is characterized as at any rate $25 \%$ of absolute sleep time with hypercapnia $(\mathrm{PaCO} 2.50 \mathrm{~mm} \mathrm{Hg})$ related with at any rate 1 of the 
accompanying: (1) snoring,

On the other hand, OSA can be analyzed if the PSG shows an event of obstructive hypoventilation, which is characterized as at any rate $25 \%$ of absolute sleep time with hypercapnia $(\mathrm{PaCO} 2.50 \mathrm{~mm} \mathrm{Hg})$ related with at any rate 1 of the accompanying: (1) snoring, (2) flattening of the inspiratory nasal pressure waveform, or (3) paradoxic thoracoabdominal motion [5].

\section{TYPES OF ORAL APPLIANCES}

Oral machines (OAs) are very much endured in many patients. Most patients favour treatment with an OA over medical procedure or CPAP, and OA consistence lies supposedly $40 \%$ to $80 \%$. Compared to OAs, CPAP yields better PSG results, particularly in decreasing AHI. CPAP is more compelling for improving sleep disordered breathing. As per the AASM practice boundaries, OA is the favoured treatment for OSA, regardless of severity, when CPAP is inadequate.
Different OA and plans have been utilized to treat OSA. The two most normal plans are tongue stabilising devices and orthodontic or mandibular advancement devices.

\section{MANDIBULAR ADVANCEMENT OA:}

Among the different sorts of OA gadgets, the mandibular advancing $\mathrm{OAs}$ is the most broadly utilized and acknowledged helpful methodology for patients with OSA. Mandibular advancing OAs extend the upper airway route by repositioning the mandible anteriorly, along these lines prevent obstruction during sleep. Mandibular advancing OAs

(Figure 1, 2) can be either fixed (i.e., the level of mandibular progression can't be changed) or semi-fixed (i.e., the level of mandibular progression can be changed). Fixed OAs have a moderately high risk of TMJ issue and dental/occlusal changes, in spite of the fact that they give a dependable restorative impact. Then again, semi-fixed OAs are accounted for as both compelling and agreeable appliance that grant slight mouth opening and lateral jaw development.

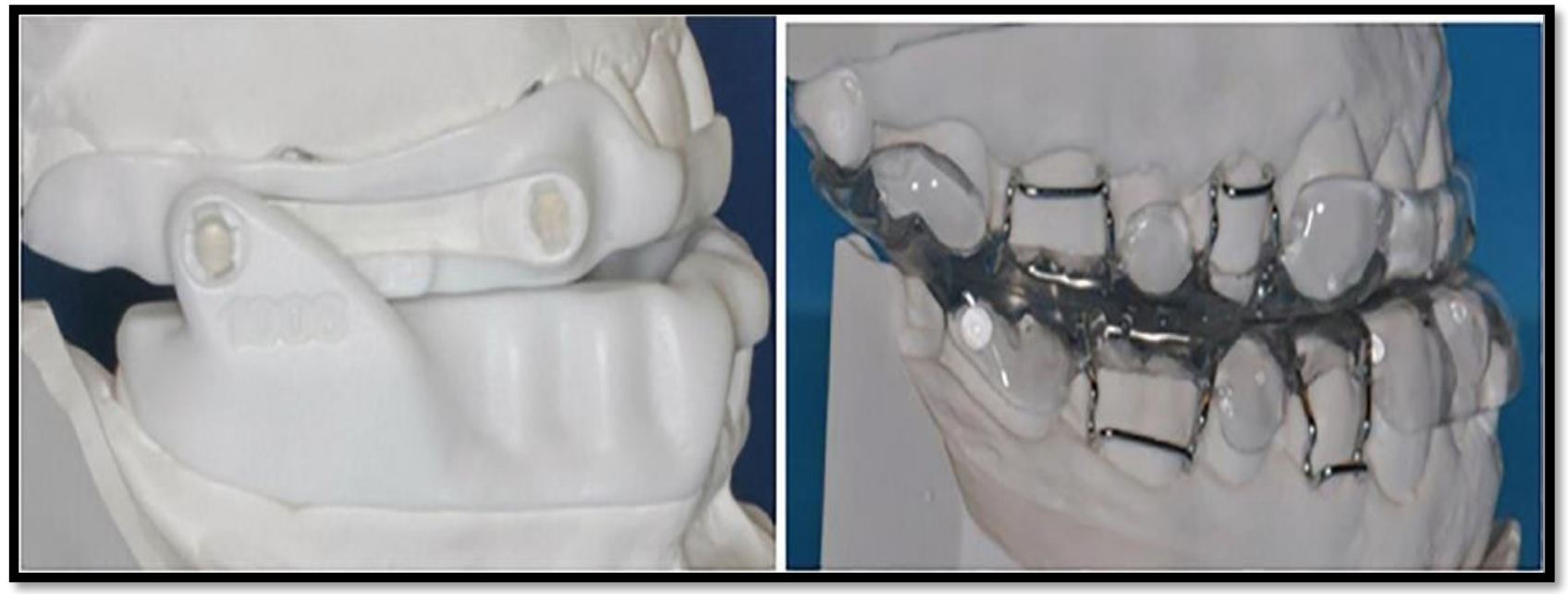

Figure 1: Mandibular Advancement Oral Appliance- Bibloc

A wide variety of various designs of the OA are fabricated today, however there are primary types, specifically the monobloc and the bibloc equipment. The former is a strong block of acrylic retained with clasps at the tooth and maintains the jaws in a set closed protruded position. The tongue stabilising bibloc has separate structures for the upper and lower jaws, prepared with connectors that protrude the mandible. The bibloc equipment permits the dentist to modify the mandibular protrusion chairside, at the same time as the monobloc equipment calls for the help of a dental technician.
It is consequently assumed that the bibloc equipment need to take much less time to fit [9].

The models utilized hard monobloc, soft monobloc and split appliances, with a same purpose: to propel the mandible around $75 \%$ of maximal projection. They are generally titratable; the monobloc by cutting it into equal parts and afterward fixed together in a more projected position. The choice of model relies upon patient and dentist's preference. The upper and lower individuals from the apparatus are associated by means of a customizable screw gathering, which fits in the palatal vault and allows titration in $0.25 \mathrm{~mm}$ 
increases. The initial mandibular progression was set at twothird of most extreme mandibular advancement, and afterward further advancements were suggested by $0.25 \mathrm{~mm}$ increments until self-reported resolution of snoring and daytime sleepiness symptoms, or until uncomfortable for the patient. Improvement in OSA was then frequently confirmed by a subsequent short-term rest concentrate with the appliance.

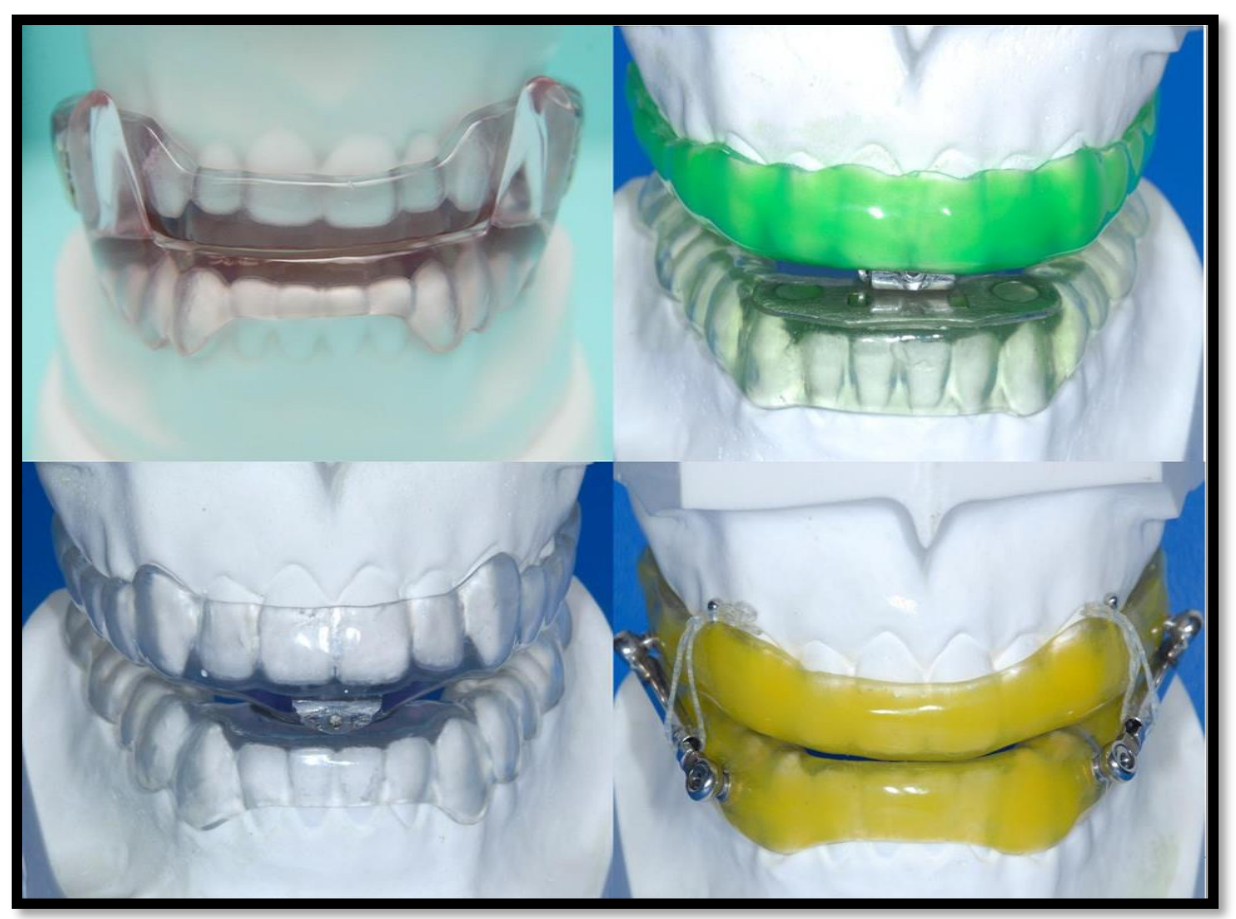

Figure 2: Mandibular Advancement Oral Appliance- Monobloc

Interestingly, in a review investigation of 805 patients, Lettieri et al. discovered a higher treatment success rate with flexible as compared to fixed devices. Serra-Torres et al. deduced in a methodical audit that adjustable mandibular advancement devices (for example biblocs) delivered preferred outcomes over fixed appliances (for example monoblocs).

Both the monobloc and bibloc appliances altogether decreased the AHI by a mean of around $12-14$ incidents each hour. The two appliances diminished the RDI value by 9 and 11 occasions each hour, separately. In a hybrid report, Bloch et al. assessed a monobloc and a Herbst appliance in moderate OSA patients. Albeit the decrease of AHI was comparable, with $14-15$ events each hour, the patients favored the monobloc to the Herbst design [10].

\section{TWIN BLOCK DEVICE}

The experimental mandibular advancement device Twin block (TB) (figure 3) has been modified for use in this situation. It consisted of two parts, one for the upper arch and the other for the lower arch. It was manufactured from selfcuring acrylic resin with occlusal covering of all teeth to minimise changes in tooth positioning that may result from its use. On average, those slopes developed an incisal opening of $8 \mathrm{~mm}$. To facilitate the retention of these devices, four Adams clasps were mounted bilaterally in the upper arch (two on the canines and two on the first molars). In the absence of the said teeth, these clasps could be displaced. Two Adams clasps were mounted bilaterally on the first premolars of the lower arch with extensions to the canines with welded hooks. These clasps, like those on the upper arch, could be moved if the teeth were missing. To ensure that the mandible remained in advanced position during sleep, the Adams clasps were attached to the upper canines with the extension hook on the lower clasp. The elastic was of medium strength, 3/16 inch in bulk. 


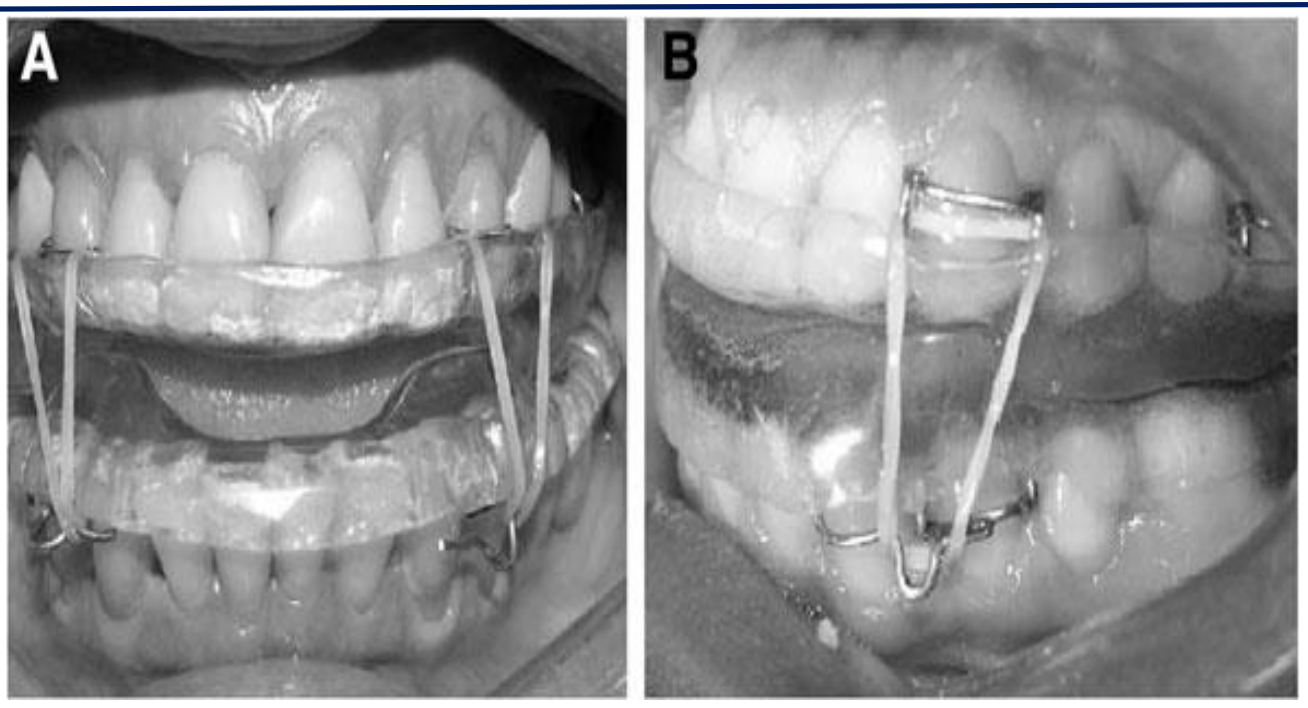

Figure 3: Twin block

\section{TONGUE STABLIZING DEVICE}

For TSDs, the best indicator of success of this appliance is a single site airway block in supine dependent OSA. On MRI, TSD responders showed a more prominent increment with TSD in AP diameter, minimum and mean CSA, and volume compared to non-responders. TSDs do not rely on dentition for retention and can be recommended for edentulous or deficiently dentate patients. TSDs can be utilized in those patients who do not respond to MAD and who need OA treatment. TSD results excess salivation, drooling, dry mouth, and soft tissue irritation.

TSDs have fairly different anatomic consequences for the airway contrasted to MADs, combining the treatments utilizing a novel hybrid appliance brought about augmentative treatment impacts. Tongue suction with $6 \mathrm{~mm}$ mandibular distension delivered preferred treatment reaction over $8 \mathrm{~mm}$ mandibular projection alone. Mix treatment may improve OA treatment reaction and demonstrate valuable results in patients with restricted mandibular distension.

\section{RAPID MAXILLARY EXPANSION (RME)}

The RME system (Figure 4), effectively performed for a long time in clinical practice, utilizes an orthodontic fixed appliance with an expansion screw, secured on chosen teeth. As per the phase of development of the teeth, the device is constructed using the first molars and permanent premolars as anchor teeth, whereas in the deciduous dentition, the second primary molars are selected, provided that they offer the stability required for appropriate treatment.

The appliance is comprised of a central expansion screw with
4 arms: 2 front arms and 2 back arms of length $1.5 \mathrm{~mm}$. Two kinds of screw are utilized: the Leone A0620/13 (Italy) and the Forestadent 1671326L (Germany); palatal split screw type "S" is utilized for particularly restricted palates. The gadget should fulfill some central contruction rules. The force is applied through the anchor teeth to act directly on the suture site, with no undesired tipping of the teeth. This application delivers a transpalatal force that surpasses physiologic levels that could create orthodontic movement. Simultaneously, the midpalatal sutures opens and orthopedic movement of the maxillae happens.

\section{ACTIVATION SYSTEM}

The screw activation device the authors use works as follows: - On the first day, morning and evening, three consecutive activations are carried out at 10-minute intervals.

- From the second one day onwards, most effective 1 activation is carried out each morning and evening.

\section{CAUSES OF DISCONTINUATION}

One of the fundamental reason for halting OA treatment was that the OA was "troublesome to use". Another fundamental driver for halting OA treatment was that patients felt that the OA had "next to zero impact" in forestalling sleep apnea. Regular difficulties of OA treatment, like dental as well as gingival discomfort or pain, or trouble sleeping, were additionally indicated as the essential driver of cessation by certain patients. The most common complication found in past investigation into $\mathrm{OA}$ treatment were tooth and gingival discomfort, dry mouth, temporomandibular and myofascial dysfunction and overabundant salivation [11]. 


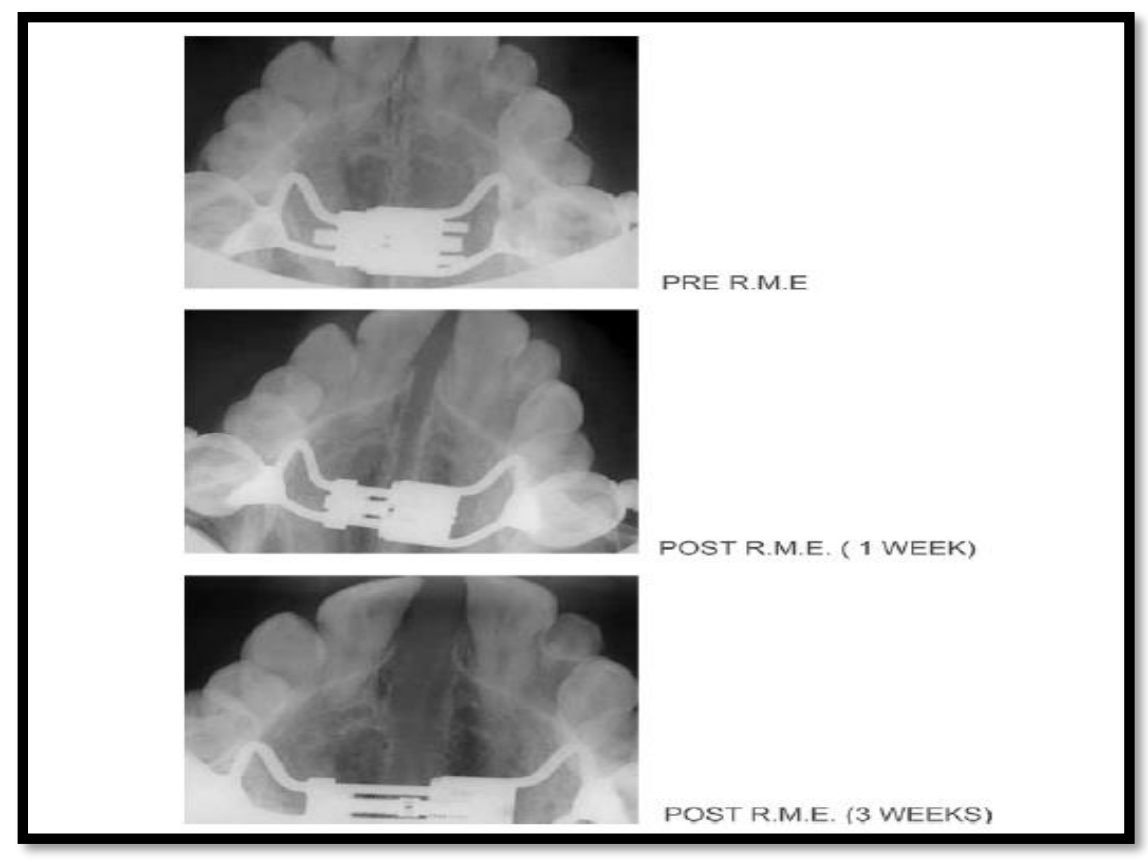

Figure 4: Rapid Maxillary Expansion

\section{References}

1. Punjabi NM. (2008) The epidemiology of adult obstructive sleep apnea. Proceedings of the American Thoracic Society. 5(2): 136-143.

2. Jonas DE, Amick HR, Feltner C, Weber RP, Arvanitis M, et al. (2017) Screening for obstructive sleep apnea in adults: an evidence review for the US Preventive Services Task Force. 317(4): 415-433.

3. Ruoff CM, Guilleminault C. (2011) Orthodontics and sleep-disordered breathing. 16(2): 271-273.

4. Nerfeldt P, Friberg D. (2016) Effectiveness of oral appliances in obstructive sleep apnea with respiratory arousals. Journal of Clinical Sleep Medicine. 12(8): 1159-1165.

5. Behrents RG, Shelgikar AV, Conley RS, Flores-Mir C, Hans M, et al. (2019) Obstructive sleep apnea and orthodontics: an American Association of Orthodontists white paper. American Journal of Orthodontics and Dentofacial Orthopedics. 156(1): 13-28.

6. Adult Obstructive Sleep Apnea Task Force of the American Academy of Sleep Medicine. (2009) Clinical guideline for the evaluation, management and long-term care of obstructive sleep apnea in adults. Journal of clinical sleep medicine. 5(3): 263276.

7. Punjabi NM. (2016) COUNTERPOINT: is the apnea-hypopnea index the best way to quantify the severity of sleep-disordered breathing? No. Chest. 149(1): 16-19.

8. Rapoport DM. (2016) POINT: Is the apneahypopnea index the best way to quantify the severity of sleep-disordered breathing? Yes. Chest. 149(1): 14-16.

9. Isacsson G, Fodor C, Sturebrand M. (2017) Obstructive sleep apnea treated with custom-made bibloc and monobloc oral appliances: a retrospective comparative study. Sleep and breathing. 21(1): 93 100.

10. Pirelli P, Saponara M, De Rosa C, Fanucci E. (2010) Orthodontics and obstructive sleep apnea in children. Medical Clinics. 94(3): 517-529.

11. Nishigawa K, Hayama R, Matsuka Y. (2017) Complication causing patients to discontinue using oral appliances for treatment of obstructive sleep apnea. Journal of Prosthodontic Research. 61(2): 133-138. 